\title{
Proceso de activización de misereo(r), contemplo(r) y palpo(r): estudio diacrónico*
}

\author{
Activization process of misereo(r), contemplo( $r)$ and palpo(r): \\ diachronic study
}

Francisca Toro Varela**

\section{RESUMEN}

El trabajo se centra en la posible relación entre las funciones sintácticas de verbos deponentes de experiencia que se someten diacrónicamente a un proceso de activización y algún cambio en su continuum de transitividad. El objetivo del estudio consistió en determinar la influencia de la transitividad de la construcción sintáctica en el proceso de activización de los verbos misereor, contemplor y palpor. Mediante un trabajo de corpus, se identificó la codificación casual de los roles semánticos de Experimentador y Estímulo, tanto de deponentes activizados como de deponentia tantum, en latín preclásico y clásico. Se concluye que existe una tendencia diacrónica a transitivizar en los deponentes activizados, en tanto surge en el período clásico una nueva restricción para las estructuras intransitivas: no pueden aparecer con las formas activas de tales verbos, corroborando así la existencia de un periodo de inestabilidad en el latín temprano que habilita el paso para el asentamiento de la activización en el latín tardío.

\section{ABSTRACT}

The article focuses on the possible relationship between the syntactic functions of experiential deponent verbs. The latter diachronically undergo a reform process and some change in
Palabras clave: activización, deponentes, transitividad, verbos de experiencia.
Keywords:

Reform, Deponents,

\footnotetext{
* Este artículo se desprende de mi trabajo final de Magíster (2018), financiado por la Agencia Nacional de Investigación y Desarrollo, ANID/Magíster Nacional/2016-22160224.

** Chilena. Estudiante de doctorado de la Pontificia Universidad Católica de Chile. Profesora en la Universidad de Santiago de Chile, Santiago, Chile.fptoro@uc.cl
} 
their transitivity continuum. This research aims to determine how syntactic construction's transitivity influences the reform process of the verbs misereor, contemplor, and palpor. Through a corpus work of Latin texts, the casual encoding of the semantic roles of Experiencer and Stimulus was identified, both from reformed deponents and deponentia tantum in Preclassical and Classical Latin. In conclusion, the reformed deponents tend to diachronically become more transitive since a new restriction for the intransitive structures arises in Classical Latin: they cannot appear with such verbs' active formations. This corroborates an instability period in Early Latin, which provides the path to establish the reform in Late Latin.
Transitivity, Experiential verbs 


\section{Introducción}

\section{Contextualización y estado de la cuestión}

Si bien el término deponente se define tradicionalmente a partir de la idea de carencia implícita en su definición etimológica (Lat. dēpōnere 'dejar caer'), actualmente puede ser tratado como una feature mismatch (Baerman, 2007), en tanto su morfología no se corresponde con su comportamiento sintáctico. Como indica Grestenberger (2014), "in an active non-active voice system, a deponent is [a voice mismatch verb] syntactically active and transitive but takes non-active morphology" (p. 12).

Uno de los procesos verbales que tuvo lugar ampliamente en el periodo tardío del latín fue el de activización de deponentes. Aquí los verbos fueron flexionados como activos, manteniendo así dos variantes, la activa y la deponente, y que comparten en principio las mismas propiedades semánticas y sintácticas (Pinkster, 2015). Este proceso afectó a deponentes de diferentes campos semánticos y fue aumentando progresivamente con el tiempo. Según Flobert (1975), se observa un incremento de verbos activizados desde el latín preclásico (21\%) hacia el latín clásico (25\%), tendencia que se marcó aún más hacia los periodos más tardíos del latín (77\%).

Existen varias explicaciones al respecto: por una parte, la existencia de deponentes con forma en - $o$ y en -or se justifica en tanto estas formas representan el comienzo de un proceso de cambio de nivel, en el que intentan parecerse al paradigma activo y las dos formas compiten entre sí (Baldi, 1973). Por otra parte, se ha tratado de justificar a partir del carácter medio que conservan los deponentes en su marca $-r$, lo cual respondería a una elección individual, probablemente estilística del autor y no a una característica semántica (Baldi, 1973). Lo que es claro, al menos, es que las activizaciones no se deben a una causa única, sino que existen varios factores que influyen, como presiones paradigmáticas y semánticas, transitividad, banalización y elecciones individuales de los autores (Flobert, 1975).

Un aspecto interesante de la deponencia es que en muchas lenguas indoeuropeas ${ }^{1}$ un número importante de verbos pertenecientes al do-

Existen verbos deponentes en otras lenguas indoeuropeas con sistema de voz "tipo griego". De las antiguas, hitita, tocario, védico, avéstico, griego, irlandés antiguo; de las modernas, griego y albanés (Grestenberger, 2015; Baerman, 2007). 
minio de la experiencia existen preponderantemente $-\mathrm{y}$, a veces, exclusivamente- en voz media (deponentes o media tantum), mientras que los verbos agentivos tienden a realizarse con formas activas o medias, lo que parecería sugerir que la morfología del verbo representa una marca distintiva para diferenciar verbos experienciales de verbos agentivos (Dahl \& Fedriani, 2012). Esto podría explicar que muchos verbos experienciales en latín sean deponentes ${ }^{2}$.

A diferencia de los deponentes agentivos - sobre los que resultaría razonable que, debido a la similitud semántica con los verbos activos, se asemejaran también a ellos en su morfología-, los deponentes experienciales son inherentemente menos transitivos que los verbos agentivos (Dahl \& Fedriani, 2012), pues se caracterizan por poseer sujetos que desempeñan un rol de Experimentador que, si bien comparten rasgos semánticos con el Agente (como el rasgo de humanidad o animacidad), se caracterizan por poseer los rasgos de menor voluntad y control (Verhoeven, 2007) que los Pacientes, Recipientes y Beneficiarios (Luraghi, 2010). En este sentido, en tanto un verbo experiencial exhibe rasgos más pacientivos y, por tanto, más parecidos a los verbos medios y deponentes, es que la activización resulta menos justificable en este tipo de verbos.

A partir del estudio de Flobert (1975), si bien el proceso de activización se desarrolla con fuerza en el latín tardío, también es posible encontrar casos de activizaciones en épocas anteriores. Es esperable, por tanto, que en estos primeros estadios del latín hayan existido ciertos patrones lingüísticos que habilitaran el cambio de morfología no-activa a activa de estos verbos, especialmente aquellos patrones que involucran un cambio en su grado de transitividad, puesto que si tradicionalmente se ha asociado la deponencia con rasgos de menor transitividad, se puede suponer entonces que un cambio en el continuum de transitividad de un verbo podría condicionar otro tipo de cambio.

2 De los deponentes que fue posible listar a partir del chequeo bibliográfico (Dahl \& Fedriani, 2012; Fedriani, 2014; Flobert, 1975) encontramos 37 verbos experienciales (abominor, admiror, adueneror, aemulor, arbitror, aspernor, comminiscor, commiseror, compatior, conqueror, conspicor, contemplor, defetiscor, delector, despicor, gratulor, intueor, irascor, laetor, meditor, miror, misereor, miseror, morior, nascor, obliuiscor, opinor, orior, palpor, patior, queror, recordor, reminiscor, reor, stomachor, suspicor y uereor), de los cuales 19 despliegan doble morfología: la deponente y la activa. 
Teniendo como hipótesis de trabajo que la transitividad de los verbos deponentes experienciales incide en su proceso de activización, el objetivo de este artículo fue determinar la influencia de la transitividad de la construcción sintáctica (esto es, codificación de los roles semánticos de Experimentador y Estímulo) en el proceso de activización de los verbos misereor, contemplor y palpor en latín preclásico y clásico.

\section{Fundamentación teórica: la noción de transitividad y el modelo de la gramática de construcciones}

El término transitividad ha sido bastante discutido en la literatura, pues no es un tema que esté del todo resuelto. Tradicionalmente, los verbos transitivos corresponden a verbos divalentes (o trivalentes), que requieren de un complemento directo para adquirir suficiencia significativa, mientras que los verbos intransitivos son esencialmente monovalentes y prescindirían de su objeto (García-Hernández, 1990; Luraghi \& Parodi, 2008; Næss, 2007; Tsunoda, 1999). Sin embargo, se trata de definiciones bastante problemáticas (Torrego, 2010), especialmente cuando se las intenta aplicar a otras lenguas: en efecto, las propiedades semánticas del verbo transitivo no se codifican siempre en las lenguas con un objeto acusativo y, al contrario, no siempre que exista un objeto acusativo se tiene necesariamente una construcción semánticamente transitiva (Baños, 2009, p. 384). Esto hace que sea un concepto muy complejo ${ }^{3}$.

Propuestas como las de Hopper \& Thompson (1980) han aportado a la discusión. Ellos presentan la transitividad como una escala que fluctúa entre los polos de baja y alta transitividad, de acuerdo con una serie de parámetros o propiedades semánticas. De esta manera, más que una propiedad intrínseca del verbo, la transitividad comprende un conjunto de variables aplicables al evento verbal completo ${ }^{4}$, pues no son los verbos los que pueden ser más o menos transitivos, sino la predicación completa (Luraghi \& Parodi, 2008).

Para una perspectiva interlingüística, ver Næss 2007, p. 12.

No ha de confundirse con el concepto de "valencia", que sí corresponde a una propiedad del verbo (Næss 2007). 
Siguiendo propuestas como la de Næss (2007), en esta investigación se entienden los roles en términos de prototipos (proto-roles) y no como categorías discretas, para lo cual se distinguen un protoAgente y un proto-Paciente distribuidos dentro de un continuum $^{5}$ : el proto-Agente codificado como el sujeto del predicado activo, mientras que el proto-Paciente se lexicaliza como un objeto directo (Dowty, 1991).

Ahora bien, para las lenguas indoeuropeas, el sujeto de una construcción se lexicaliza prototípicamente en caso nominativo y el objeto directo como un acusativo. Sin embargo, no todos los sujetos nominativos y los acusativos objeto poseen los rasgos de proto-Agente y protoPaciente en el sentido de Dowty (1991), pues debe considerarse que la transitividad ha estado ligada tradicionalmente a las nociones de agentividad y control (Dahl \& Fedriani, 2012), de las que depende esta codificación de roles y, además, las funciones sintácticas de sujeto-objeto no se lexicalizan siempre en el latín como estructuras nominativoacusativo, sino que así como ocurre en otras lenguas indoeuropeas, tales argumentos pueden estar codificados en otros casos. Es muy frecuente, por ejemplo, encontrar objetos en caso genitivo o dativo, y no por ello es inválida la interpretación transitiva de la construcción: "lo importante es que se trata del primer complemento requerido por el verbo" (García-Hernández, 1990, p. 3).

Lo que sí varía, empero, es su grado de transitividad dentro del continuum. En efecto, el caso acusativo puede calificarse con mayor afección y, por tanto, como más alto dentro del continuum de transitividad, mientras que la selección de casos oblicuos refleja una tendencia interlingüística de menos afección o transitividad reducida ${ }^{6}$ (Dahl \& Fedriani, 2012). En este sentido, no es necesariamente intransitiva una construcción que contiene un caso oblicuo como argumento sintáctico (como propondría una teoría tradicional de transitividad) (Tsunoda, 1985; 1999). Incluso si no existe un segundo argumento sintáctico

\footnotetext{
5 A partir de una serie de propiedades para los proto-roles de Agente y Paciente (Dowty 1991).

$6 \quad$ Especialmente el dativo. Tómese en cuenta que esto es particularmente relevante si se considera que el estudio de Dahl y Fedriani (2012) trata de verbos de experiencia (calificados como inherentemente menos transitivos que los verbos agentivos) y con morfología de la voz media.
} 
es posible valorar la cláusula como transitiva, siempre y cuando el elemento argumental faltante pueda ser recuperado como objeto nulo ${ }^{7}$. Esto es particularmente importante para las lenguas sintéticas, que tienen la característica de presentar objetos nulos, es decir, de omitir el objeto sintáctico y recuperarlo semánticamente al ser altamente predecible por contexto (Luraghi, 2004) ${ }^{8}$.

Desde el modelo de la gramática de construcciones, los objetos nulos son considerados parte de la construcción, pues este enfoque le otorga un valor muy prominente a la dimensión semántica, en tanto concibe los cambios no como propios de la gramática, sino como modificaciones basadas en el uso (Traugott, 2008). De esta manera, se entenderá construcción como parte de un continuum léxico-sintáctico y no como categorías totalmente discretas (Hoffmann \& Trousdale, 2013). La ventaja de esta aproximación teórica es que permite entender los diversos cambios de la lengua de manera diferenciada a como ha sido entendida tradicionalmente, es decir, sin separar semántica de sintaxis, además de considerar ciertos marcadores pragmáticos como parte de la gramática (Traugott, 2015).

Por otra parte, en cuanto a los distintos cambios que ocurren dentro del sistema de una lengua, el concepto de construccionalización permite relacionar el fenómeno de activización con un proceso de creación de pares nuevos de forma y significado (formnew-meaningnew pairing), que afectan su esquematicidad, productividad y composicionalidad (Traugott 2015). En consecuencia, y para efectos de este estudio, se entenderá una construcción transitiva como la selección de un segundo argumento (explícito o no) y construcción intransitiva como la ausencia de este, sin posibilidad de recuperarlo contextualmente como objeto nulo.

\section{Método de investigación}

El corpus fue tomado de la base de datos Library of Latin Texts - Series A (LLT-A) [en línea]. Se seleccionaron dos periodos dentro de la categoría Antiquitas: el periodo arcaico o preclásico (siglos III a.C. y II a.C.)

Sobre objetos nulos en latín, ver Luraghi 2004.

Como en otras lenguas, los objetos nulos son muy frecuentes en latín, especialmente en los verbos de experiencia, que se usan muchas veces sin la aparición explícita del Estímulo (Fedriani, 2014). 
y el periodo clásico (siglos I a.C. y I d.C.). Como autores del latín preclásico se encuentran Plauto, Ennio, Terencio, Catón, Accio y Nevio. Del periodo clásico se estudiaron los siguientes autores: Catulo, Cicerón, Salustio, Virgilio, Lucrecio, Horacio, Tito Livio, Ovidio, Lucano, Titinio, Pomponio, Plinio, Valerio Máximo, Columela, Séneca el joven, Séneca el viejo, Apuleyo, Lucilio, Manilio, Estacio, Juvenal, Pacuvio, Turpilio, Petronio, Propercio, Publilio, Q. C. Quadrigario, Cornelio Nepote, Cornelio Tácito, Veleyo Patérculo, Silio Itálico, Quintiliano y Valerio Flaco.

En cuanto a la muestra, se clasificaron los 37 verbos deponentes experienciales en verbos deponentes activizados (grupo experimental) y verbos deponentes no-activizados (deponentia tantum, grupo control) para contrastar, por un lado, el comportamiento de los verbos activizados con su contraparte deponente (análisis intragrupal) y, por otro, el comportamiento de los verbos activizados con otros verbos de experiencia que solo funcionan como deponente (análisis intergrupal). Se los clasificó, además, según los subdominios semánticos de Verhoeven (2007): como verbos de emoción, percepción, cognición, sensación corporal o volición.

Como criterios morfológicos, se buscaron todas las formas verbales, incluyendo infinitivos, subjuntivos e imperativos. Se descartaron, empero, las formas de gerundio, supino y participio por la dificultad de identificarlas claramente con una morfología activa o pasiva. Se consideraron, además, las variantes gráficas de los verbos.

Debido a razones metodológicas, se seleccionaron los verbos que registraran, al menos, una coincidencia con morfología activa en cada uno de los periodos estudiados. En cuanto a los testimonios del corpus, se consideraron los verbos que aparecieran en autores reconocidos, en todos los géneros literarios disponibles y en textos idealmente completos, con el fin de acceder más fácilmente al contexto. Así, se descartaron, por un lado, los verbos que no tuvieran realizaciones activas en alguno de los dos periodos y, por el otro, textos demasiado fragmentarios.

Tras estas restricciones y consideraciones, quedaron solo tres verbos activizados ${ }^{9}$ : misereor, contemplor y palpor, divididos entre verbos 
de emoción y percepción ${ }^{10}$. La muestra de este grupo quedó conformada por 292 formas verbales: 222 casos de misereor (verbo de emoción) y 70 entre contemplor y palpor (verbos de percepción).

Como deponentia tantum (grupo control), se tomaron los verbos deponentes correspondientes a los mismos grupos semánticos que los tres activizados. En este caso, cinco verbos deponentes de emoción (uereor, aspernor, laetor, queror, admiror) y dos de percepción (conspicor, intueor), priorizando las mismas formas verbales y los mismos autores que aparecieron con los verbos activizados. No obstante, debido a que los casos resultaron ser excesivos (1.500 casos en total) se hizo una selección aleatoria. La muestra final de estos verbos fue de 287 casos: 217 verbos de emoción y 70 verbos de percepción.

Finalmente, el análisis consistió en la identificación y tabulación de los datos de codificación de caso de los roles de Experimentador y Estímulo. Luego, se determinó el grado de transitividad de la construcción según la selección de casos acusativo, genitivo y dativo como prueba de construcción transitiva (además de las construcciones de ne/quod + subjuntivo), y la no-selección de estos casos como prueba de intransitivación. Por último, se valoraron los datos obtenidos a través de la prueba estadística paramétrica de regresión logística con efectos de interacción (ANOVA) en el programa R-Studio.

\section{Análisis y resultados}

\subsection{Misereor, contemplor y palpor en periodo preclásico}

Durante el latín preclásico es posible reconocer tanto construcciones transitivas como intransitivas en los tres verbos activizados. El verbo de emoción misereor aparece construido con EXPNOM-STIMGEN ${ }^{11}(1)$, mientras que la construcción de misereo es la impersonal EXPACC y STIMGEN (1b), que solo se presenta en este periodo con la forma activizada

arcaico y clásico (con escasas ocurrencias y, en varios casos, en textos fragmentarios). Esto explica que solo tres verbos estuvieran habilitados para su análisis.

10 Consideramos "emoción" a aquellos estados o reacciones que tengan un correlato en el ámbito biológico (tanto psicológico como fisiológico) y "percepción" a aquello que simplemente se recibe mediante los cinco sentidos básicos mencionados.

${ }_{11}$ Se utilizarán las abreviaturas de Dahl y Fedriani (2012): EXP = Experimentador, STIM = Estímulo. 
y no la deponente. Por su parte, los verbos de percepción contemplor (2a) y contemplo (2b) se construyen ambos con EXPNOM-STIMACC, construcción que toma también la variante activizada de palpor (3a), pero no así el deponente, que selecciona en este periodo EXPNOM-STIMDAT (3b).

(1a) Sed sine argento frustra es qui me tui misereri postulas ("Pero sin dinero es inútil que me pidas que me apiade de ti”. Pl., Pse. 378).

(1b) Neminis miserere certumst, quia mei miseret neminem ("Estoy decidido a no tener compasión de nadie, ya que nadie se compadece de mí". Pl., Capt. 765).

(2a) Loquimini mecum, Antipho, contemplamini me ("Hablad conmigo, Antifón, miradme bien". Ter., Phor. 550).

(2b) Edepol, qui quom hanc magis contemplo, magis placet ("Te juro, por Pólux, que cuanto más la contemplo, más me gusta”. Pl., Pers. 564).

(3a) Nimis hic scitust sycophnata qui quidem meus sit pater obseruatote, quam blande mulieri palpabitur ("iVaya hábil impostor que está hecho mi padre! Ya veréis con qué suavidad va a calmar a la señora". Pl., Amph. 507).

(3b) Quid faciam? AG. Exora, blandire, palpa (“¿Qué puedo hacer? AG. Suplícale, halágala, acaríciala”. Pl., Poen. 357).

Hay varios aspectos que deben tenerse en cuenta: en el ejemplo (4), la construcción de acusativo con infinitivo (AcI), me misereri postulas, equivale a una construcción nominativa con verbo conjugado que se puede permutar por la cláusula subjuntiva ut ego tui miserear postulas (EXPNOM-STIMGEN). En el caso (2a), al estar en modo imperativo, no aparece explícita la codificación de Experimentador, sino que basta solo con el vocativo Antipho. En el ejemplo (3a), es posible recuperar un nominativo a partir del pater de la cláusula anterior. Desde el punto de vista de la gramática de construcciones, en el ejemplo ( $3 b$ ) el contexto permite restituir un argumento acusativo implícito, pues en esta parte de la obra se encuentran dos personajes que conversan acerca de cómo aplacar el enojo de cierta muchacha. Probablemente los verbos exora, blandire y palpa han seleccionado el pronombre acusativo illam de la cláusula anterior.

Por otra parte, además de la codificación casual de los roles de Experimentador y Estímulo, también existen casos en que el Estímulo no se codifica sintácticamente ni puede ser recuperado como objeto nulo, proyectando una construcción intransitiva. Los verbos que presentan esta estructura fueron 10: los deponentes contemplor (4a) y palpor (4b) y las formas activizadas misereo (4c) y contemplo (4d): 
(4a) Attat, singulum video vestigium, sed is hac abiit. Contemplabor (“¡Caramba! Solo veo las huellas de uno. Pero este se fue por aquí. Observaré con atención”. Pl., Cist. 702).

(4b) Hoc sis uide, ut palpatur: nullust, quando occepit, blandior ("Mira esto, por favor, qué bien sabe engatusar: no hay nadie más halagador cuando se lo propone". Pl., Merc. 169).

(4c) Ita me di bene ament, sapienter: atque equidem miseret tamen ("Así los dioses me favorezcan como es cierto que ha hablado inteligentemente; y, sin embargo, ciertamente me compadezco". Pl., Pers. 639).

(4d) Tu cetera cura et contempla et de meis uenator uerbis ("Encárgate tú del resto, estate atento y sigue la pista que te indiquen mis palabras". Pl., Mil. Glor. 1029).

\subsection{Misereor, contemplor y palpor en periodo clásico}

Las construcciones transitivas de este periodo se codifican del mismo modo que en la época preclásica: misereor con EXPNOM-STIMGEN, contemplor con EXPNOM-STIMACC y palpor con EXPNOM-STIMDAT. Por su parte, solo los verbos misereor y contemplor presentan construcciones intransitivas en este periodo, similares a las del latín preclásico.

Sin embargo, a diferencia del comportamiento sintáctico del periodo preclásico, las activizaciones de esta época solo aparecen en construcciones transitivas: con la estructura EXPACC-STIMGEN en misereo (5a) y con EXPNOM-STIMAcC tanto en contemplo (5b) como en palpo (5c). Es importante enfatizar que la diferencia sintáctica que mostraron los verbos palpor y misereor y sus variantes activizadas en el periodo anterior se replica en el comportamiento presente en este periodo.

(5a) Miseret me eorum, qui sine frustis uentrem frustrarunt suum ("Me apiado de ellos, que sin tener ningún pedazo de alimento engañan a su estómago". Pomp., Atell. 79).

(5b) Contemplare locum caeli sedemque uagarum ("Es preciso contemplar la posición en el cielo y la colocación de los planetas". Manil., Astr. 2.644).

(5c) Manibusque cruentis palpat inane solum saeuaque ita uoce precatur ("y con las manos manchadas de sangre golpea la tierra hueca, y en acentos nefastos pronuncia esta oración”. Estac., Theb. 1.55).

\subsection{Deponentia tantum: grupo control}

A diferencia de los verbos deponentes que activizan, los deponentia tantum muestran un comportamiento regular de un periodo a otro. La 
construcción transitiva prototípica EXPNOM-STIMACC aparece en los verbos admiror, uereor, aspernor, queror, conspicor e intueor en ambos periodos estudiados, mientras que laetor, en el periodo más antiguo utiliza la estructura EXPNOM-STIMABL y, en el periodo clásico, amplía su red argumental hacia objetos acusativos. Y queror, además de la construcción acusativa, adopta para el periodo clásico una estructura preposicional con ablativo ${ }^{12}$.

Por otra parte, construcciones intransitivas solo aparecen en los verbos de emoción uereor, laetor y queror en ambos periodos, aunque con menor frecuencia hacia el latín clásico.

\section{Discusión de los datos}

Respecto del tipo de construcción (transitiva o intransitiva) ${ }^{13}$ seleccionada en cada periodo, los valores porcentuales de las estructuras de los verbos de experiencia analizados han sido agrupados de manera sintética en la Tabla 1:

Tabla 1.

Distribución de construcciones transitivas e intransitivas en latín preclásico y clásico.

\begin{tabular}{lccc}
\hline & & Latín preclásico & Latín clásico \\
\hline \multirow{2}{*}{ Deponentes } & $\mathrm{T}=$ & $12(86 \%)$ & $155(80 \%)$ \\
\cline { 2 - 4 } & $\mathrm{I}=$ & $2(14 \%)$ & $38(20 \%)$ \\
\hline Activizados & $\mathrm{T}=$ & $45(85 \%)$ & $32(100 \%)$ \\
\cline { 2 - 4 } & $\mathrm{I}=$ & $8(15 \%)$ & $0 \%$ \\
\cline { 2 - 4 } deponentia tantum & $\mathrm{T}=$ & $50(85 \%)$ & $217(95 \%)$ \\
\cline { 2 - 4 } & $\mathrm{I}=$ & $9(15 \%)$ & $11(5 \%)$
\end{tabular}

Nota: $\mathrm{T}=$ transitivo, $\mathrm{I}=$ intransitivo.

Fuente: Elaboración propia.

Si bien es esperable que en el periodo clásico exista mayor cantidad de testimonios registrados en el corpus, es importante destacar que en los tres casos, es decir, tanto en verbos deponentes no-activi-

\footnotetext{
12 Si bien aún es posible encontrar un segundo argumento acusativo, la mayoría de las construcciones de queror en este periodo son cláusulas preposicionales de de + abl o estructuras de AcI.

13 La razón de considerar aquí la transitividad como valores dicotómicos permite observar las elecciones estructurales que hacen los verbos a lo largo de los siglos y, a partir de esto, esbozar una tendencia diacrónica de comportamiento.
} 
zados como en los activizados y los deponentia tantum, se mantiene la misma proporción de selección de estructuras transitivas/intransitivas en el periodo preclásico $(86 \%, 85 \%$ y $85 \%$ de construcciones transitivas, respectivamente), aunque para el periodo clásico la proporción varía, pues mientras las formas activizadas muestran un crecimiento del $15 \%$ de las estructuras transitivas y los deponentia tantum un 10\% de aumento, las formas deponentes decrecen en un $6 \%$. Es posible apreciar estos datos de manera gráfica en la Figura 1:

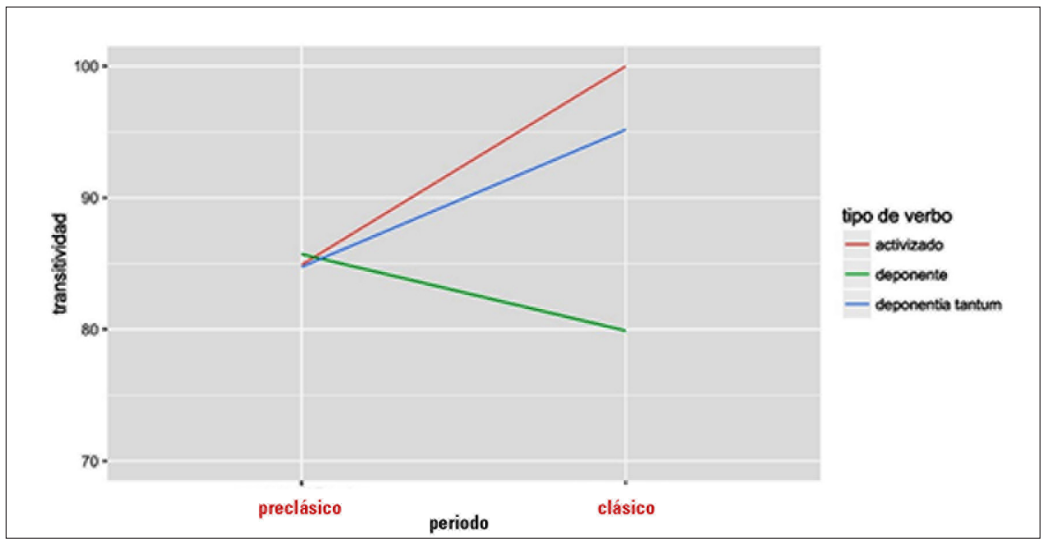

Figura 1. Frecuencia de transitividad en cuanto al periodo y tipo de verbo.

Según los datos analizados y expuestos en la Figura 1, existe una tendencia de crecimiento a lo largo de los siglos en las estructuras transitivas de los verbos activizados y deponentia tantum, lo que se relaciona significativamente $(p=0,0176)$ con el decrecimiento de las estructuras transitivas de los verbos deponentes, en desmedro de las construcciones intransitivas, ya que en la medida que la transitividad aumenta en los activizados y deponentia tantum, lo intransitivo disminuye, y lo mismo a la inversa para el caso de los deponentes: en tanto bajan las construcciones transitivas de un periodo al otro, la intransitivación aumenta.

Por otro lado, a partir de una distinción de morfología de voz, se aprecia una diferencia entre la forma deponente y la forma activa, desde el punto de vista de la transitividad de la cláusula. Como categorías discretas (transitivo o intransitivo), los deponentes que tienen una contraparte activa muestran una mayor fluctuación de un periodo al otro, respecto del tipo de estructura que emplean (Tabla 2), a diferencia de los 
verbos deponentia tantum, donde la admisión de estructuras transitivas e intransitivas ocurre de manera diacrónicamente estable: verbos que presentan cierta estructura en el periodo arcaico, la mantienen para el periodo clásico, tal como muestra la Tabla 3 .

Tabla 2.

Transitividad clausal en deponentes experienciales que activizan.

\begin{tabular}{llcc}
\hline & & Periodo arcaico & Periodo clásico \\
\hline Deponente & contemplor & $\mathrm{T} / \mathrm{I}$ & $\mathrm{T} / \mathrm{I}$ \\
\cline { 2 - 4 } & palpor & $\mathrm{T} / \mathrm{I}$ & $\mathrm{T}$ \\
\cline { 2 - 4 } & misereor & $\mathrm{T}$ & $\mathrm{T} / \mathrm{I}$ \\
\hline Activizado & contemplo & $\mathrm{T} / \mathrm{I}$ & $\mathrm{T}$ \\
\cline { 2 - 4 } & palpo & $\mathrm{T}$ & $\mathrm{T}$ \\
\cline { 2 - 4 } & misereo & $\mathrm{T} / \mathrm{I}$ & $\mathrm{T}$ \\
\hline
\end{tabular}

Nota: $\mathrm{T}=$ transitivo, $\mathrm{I}=$ intransitivo.

Fuente: Elaboración propia.

Como se ve en la Tabla 2, a medida que se reduce el tipo de construcción de un periodo al otro (como palpor), se amplía al pasar de un tipo de construcción en el primer periodo a dos en el siguiente (misereor). En cualquier caso, como resultado, se tienen tanto construcciones transitivas como intransitivas en las formas deponentes y activas en el periodo arcaico; y en el periodo clásico, en cambio, solo los deponentes con ambas construcciones, pues las formas activizadas solo se utilizan en construcciones transitivas. Este hecho, como se verá más adelante, constituye un argumento para abogar en favor de un proceso de construccionalización de las formas activizadas.

Tabla 3.

Transitividad clausal en deponentes experienciales que no activizan.

\begin{tabular}{lcc}
\hline & Periodo arcaico & Periodo clásico \\
\hline Admiror & $\mathrm{T}$ & $\mathrm{T}$ \\
\hline Uereor & $\mathrm{T} / \mathrm{I}$ & $\mathrm{T} / \mathrm{I}$ \\
\hline Aspernor & $\mathrm{T}$ & $\mathrm{T}$ \\
\hline Laetor & $\mathrm{T} / \mathrm{I}$ & $\mathrm{T} / \mathrm{I}$ \\
\hline Queror & $\mathrm{T} / \mathrm{I}$ & $\mathrm{T} / \mathrm{I}$ \\
\hline Conspicor & $\mathrm{T}$ & $\mathrm{T}$ \\
\hline Intueor & $\mathrm{T}$ & $\mathrm{T}$ \\
\hline Nota: $\mathrm{T}=$ transitivo, $\mathrm{I}=$ intransitivo. & &
\end{tabular}

Fuente: Elaboración propia. 
Desde un punto de vista sintáctico, dentro de la red argumental de los verbos de experiencia aquí estudiados, los roles de Experimentador y Estímulo son codificados de manera similar en los verbos de emoción y en los de percepción, puesto que como se ve en la Tabla 4, en general todos prefieren la estructura argumental con acusativo (EXPNOM-STIMACC), situándose inmediatamente en el extremo más transitivo del continuum de transitividad prototípica de Dowty (1991).

Tabla 4.

Estructura argumental de verbos de experiencia.

\begin{tabular}{|c|c|c|c|c|c|}
\hline & \multicolumn{4}{|c|}{ Construcciones generalizadas } & \multirow{2}{*}{$\begin{array}{c}\begin{array}{c}\text { Construcciones } \\
\text { inversas }\end{array} \\
\text { EXPACC-STIMgEN }\end{array}$} \\
\hline & $\begin{array}{l}\text { EXPNOM- } \\
\text { STIMACC }\end{array}$ & $\begin{array}{l}\text { EXPNOM- } \\
\text { STIMDAT }\end{array}$ & $\begin{array}{l}\text { EXPNOM- } \\
\text { STIMGEN }\end{array}$ & $\begin{array}{l}\text { EXPNOM- } \\
\text { STIMABL }\end{array}$ & \\
\hline \multirow[t]{6}{*}{ Emoción } & & & Misereor & & misereo \\
\hline & Admiror & & & & \\
\hline & Uereor & & & & \\
\hline & Aspernor & & & & \\
\hline & Laetor & & & laetor & \\
\hline & queror & & & & \\
\hline \multicolumn{6}{|c|}{ Percepción contemplo(r) } \\
\hline & palpo & Palpor & & & \\
\hline & conspicor & & & & \\
\hline
\end{tabular}

Fuente: Elaboración propia.

Sin embargo, existen ciertas salvedades que escapan de esta tendencia. Dentro de los verbos de emoción, uno de los aspectos relevantes que se destaca es la diferencia (morfo)sintáctica entre la forma deponente misereor y su contraparte activizada misereo. Según los datos recogidos, y en línea con los trabajos de Fedriani (2014), la selección argumental de la forma activa es propia de verbos impersonales como pudet, taedet o piget, que utilizan la tercera persona del verbo con la estructura inversa EXPacc-STIMgen, tanto en el periodo arcaico como en el periodo clásico, mientras que la forma deponente adopta la conjugación personal con la construcción EXPnom-STIMgen. 
Si bien es posible encontrar la construcción impersonal con la forma deponente ${ }^{14}$, el patrón que se habría generalizado hacia el latín posclásico es el paradigma personal con el verbo deponente. En términos puramente diacrónicos, Fedriani (2014) explica lo siguiente: "while the active form expresses the impersonal pattern, the deponent counterpart progressively specialized as personal, along the lines of a process of regularization which led the verb miseret to abandon the impersonal construction from Classical Latin onward" (p. 162).

Lo interesante de la diferencia argumental de las estructuras sintácticas de miseret y miseretur es que, tanto en los datos del corpus como en la literatura atestiguada, la construcción personal (deponente) presenta características semánticas de mayor control e intencionalidad, mientras que la construcción impersonal (activizada), en cambio, muestra menos rasgos de control. Este escenario sintáctico, por lo demás, ya aparece atestiguado en la bibliografía. Según Tur (2013), el uso de imperativo nunca ocurre con la construcción impersonal, lo que sugiere que elementos como el control o la intencionalidad del Sujeto determinan la elección entre construcción personal o impersonal ${ }^{15}$.

Sin embargo, desde un punto de vista morfológico, llama la atención que justamente esta construcción —la impersonal-, adopte la morfología activa del verbo, que es la forma que ha estado más vinculada con las nociones de agentividad y control (Dahl \& Fedriani, 2012) $\mathrm{y}$, por otra parte, que sea la forma impersonal la que haya registrado de manera significativa ( $p=0,0396)$, según los datos analizados en esta investigación, una mayor transitividad ligada al EXPACC y menor transitividad cuando se trata de EXPNOM, ya que, como puede apreciarse en la Figura 2, si bien ambas codificaciones tienden al crecimiento, el EXPACC es considerablemente mayor.

\footnotetext{
14 Aunque Fedriani (2014) reconoce un par de casos de la forma deponente en el latín temprano con la estructura inversa, se tratan más bien de textos fragmentarios, o bien, de lectura dudosa, de manera que debido a los criterios metodológicos empleados aquí, fueron descartados de este estudio.

15 "Los ejemplos en imperativo son especialmente frecuentes en los poetas, lo que posiblemente tenga que ver con el hecho de que la estructura prosódica (misěrēre) se ajusta métricamente tanto a los hexámetros dactílicos (...) como al trímetro yámbico" (Tur, 2013, p. 247).
} 


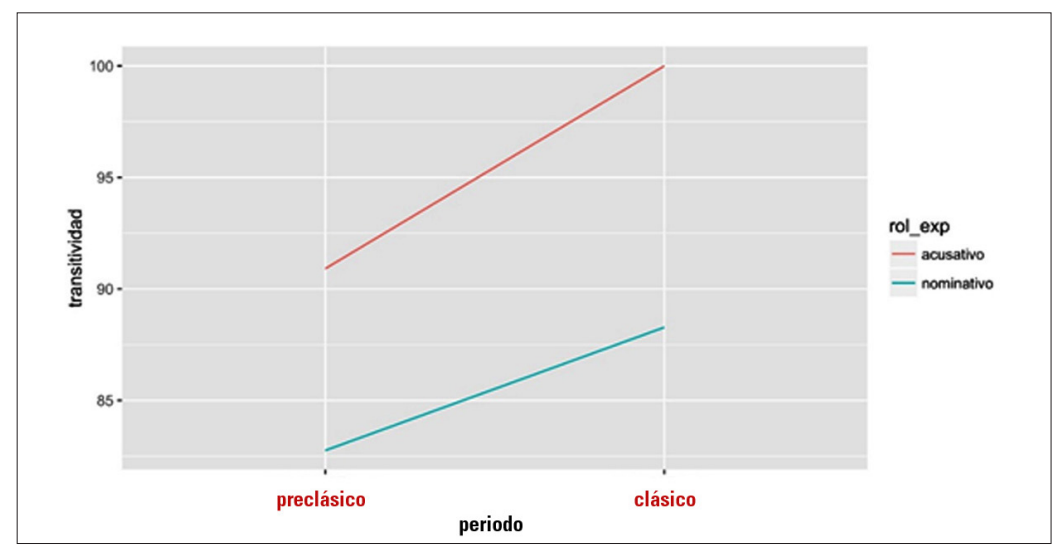

Figura 2. Frecuencia de transitividad en cuanto al periodo y rol de Experimentador. Fuente: Elaboración propia.

El hecho de que el verbo miseret se haya alineado al paradigma de verbos impersonales, cuya red argumental mostró ser significativamente más transitiva, es prueba de una alta construccionalización del esquema transitivo ya que, tomando en cuenta que los verbos impersonales son los más resistentes a aparecer en construcciones transitivas (Pieroni, 2000), cuando ocurre, se puede interpretar como reflejo de un proceso muy avanzado en su grado de transitividad. Sin embargo, la naturaleza y el comportamiento sintáctico de los verbos impersonales experienciales es un tema que escapa a este estudio.

Por su parte, dentro de los verbos de percepción, los verbos contemplor, conspicor e intueor presentan una construcción estable desde el latín preclásico al latín clásico: la construcción acusativa. Sin embargo, el verbo palpor se escapa de este patrón por medio de una diferencia en su estructura morfosintáctica, a saber: reserva la construcción acusativa para la forma activizada en los dos periodos estudiados, mientras que emplea la construcción con dativo para la forma deponente, tal como muestra la Tabla 5.

Tabla 5.

Codificación del rol de Estímulo en las formas palpor y palpo.

\begin{tabular}{lll}
\hline & Periodo arcaico & Periodo clásico \\
\hline Palpor & Dativo & Dativo \\
\hline Palpo & Acusativo & Acusativo \\
\hline
\end{tabular}

Fuente: Elaboración propia. 


\section{Conclusiones}

A partir de los resultados discutidos en el apartado anterior, es posible esbozar algunas conclusiones. En primer lugar, en cuanto a la clasificación semántica de verbos experienciales, los datos muestran que existe una diferencia significativa entre los verbos de emoción y percepción respecto de la transitividad de sus construcciones. Los verbos de percepción tienden a seleccionar construcciones más prototípicamente transitivas que los verbos de emoción, los cuales emplean otros casos además del acusativo para codificar el segundo argumento o argumento Estímulo.

En segundo lugar, los verbos misereor y palpor tienden a diferenciar la codificación de sus roles semánticos por medio de una especialización morfosintáctica. El verbo misereor solo emplea la forma activizada como una construcción impersonal y la deponente la usa como personal. Por su parte, el verbo palpor utiliza el Estímulo en dativo para la forma deponente y el Estímulo en acusativo para la forma activizada $(-r=$ STIMDAT $/-O=$ STIMACC). Esta especialización de miseret (forma activizada e impersonal) se relaciona significativamente con la mayor transitividad que muestra cuando selecciona EXPACC EN LUGAR DE EXPNOM. La especialización de palpor da cuenta del mayor grado de transitividad de la construcción activizada, pues el verbo palpo es interpretado como más prototípicamente transitivo en comparación con el deponente, en la medida que tiene como objeto un acusativo.

En tercer lugar, desde un punto de vista diacrónico, tanto los verbos que activizan como los deponentia tantum presentan un incremento estadísticamente significativo en sus estructuras transitivas en relación inversamente proporcional con las construcciones intransitivas, las que tienden a disminuir hacia el periodo clásico. Así, mientras en el periodo preclásico la proporción transitivo/intransitivo es semejante en todos los verbos de experiencia analizados, en el periodo clásico se observa una diferencia en su crecimiento, pues cuando los verbos activizados y deponentia tantum aumentan sus estructuras transitivas (con una proporción bastante similar), los verbos deponentes disminuyen las suyas y, en su lugar, suben las construcciones intransitivas.

Por otra parte, no existe una variación diacrónica en cuanto a la transitividad de los deponentia tantum, como sí la habría en aquellos 
deponentes que activizan, pues mientras la tendencia de los primeros es hacia la conservación de sus estructuras (transitiva o intransitiva), los verbos que activizan presentan no solo una alteración o inestabilidad en ellas, sino que también existe y se destaca una restricción en periodo clásico para los verbos activizados: la elección exclusiva por la estructura transitiva.

El hecho de que palpor seleccione un valor más alto en la escala de transitividad y que además, cuantitativamente, tanto palpor como misereor y contemplor acumulen más construcciones transitivas con sus morfologías activas de manera progresiva, en términos diacrónicos (resultado que logra significancia estadística), refleja que el cambio se produjo por la preferencia de un uso exclusivo basado en la relación morfología/estructura casual, lo que puede ser interpretado desde la gramática de construcciones como la acumulación gradual (progresiva en el tiempo) de ciertas restricciones contextuales de los verbos activizados.

Este hecho confirma la hipótesis inicial de la presente investigación, pues es posible afirmar que, según los datos analizados, el proceso de activización de los deponentes de experiencia está mediado por la transitividad de la construcción, dado que es posible observar una diferencia morfosintáctica entre verbos deponentes que activizan y no-activizan. En otras palabras, la transitividad que no parecería estar relacionada con la morfología del verbo en periodo preclásico, aparece vinculada en el clásico, en tanto habría una restricción de encontrar estructuras intransitivas con verbos activizados, pues las construcciones de estos verbos se habrían extendido a usos exclusivamente transitivos.

Desde la gramática de construcciones, este proceso de activización en verbos experienciales, basado en la relación entre la morfología del verbo y la disposición sintáctica de argumentos verbales, se puede interpretar como un proceso de construccionalización, en tanto una nueva morfología $(-o)$ aparece asociada con un significado particular (valor transitivo). Prueba de ello es la mayor productividad que logra el aumento en la abstracción de la nueva estructura (esquematicidad) en las etapas posclásicas del latín, debido a la escasa o nula composicionalidad que se puede ver en la flexión -o (como contempl-o) en comparación con la flexión $-r$ (contempl-o-r). Esto deriva en que la nueva 
construcción, la activizada, tenga una semántica más específica que la original (la asociación con una cierta estructura argumental).

Desde una perspectiva diacrónica, se puede concluir que dicho proceso, ampliamente masificado en el periodo posclásico (siglos II d.C. y posteriores), no fue una innovación de su tiempo, sino que es el resultado de una relación morfosintáctica, producto de un proceso de construccionalización, que comienza ya en los periodos de Plauto en el latín temprano. Según la presente investigación, por tanto, se puede sostener la existencia de un periodo de inestabilidad lingüística que constituyó una transición hacia el latín tardío y los cambios que se asentaron en ese periodo. Tal como declara Álvarez (2009), existe una tendencia a transitivizar las estructuras del latín, de modo que la desaparición de los verbos deponentes (al menos los de experiencia) hacia el periodo prerromance puede ser explicada lingüísticamente por la inclinación de la lengua a construir oraciones transitivas con verbos morfológicamente activos.

Desde el punto de vista del cambio lingüístico, este proceso de construccionalización es coherente con la IV ley de Kuryłowicz referente a la naturaleza del cambio analógico. Este principio, entendido más bien como tendencia de cambio, sostiene que cuando una evolución morfológica genera dos formas diferenciadas, la forma derivada asume la función primaria, mientras que la forma antigua se reserva para la función secundaria o reciente ${ }^{16}$ (Elvira, 1998). En el caso de la activización, la morfología en $-r$ corresponde a la forma primaria y la forma activizada - o equivale a la forma analógica. En un continuum temporal, que va desde el latín preclásico al latín clásico, la forma analógica se asocia exclusivamente con el uso más extendido de la forma primaria (uso transitivo) en periodo preclásico, y desarrolla diacrónicamente una generalización de ese marco argumentativo, tal como muestra el esquema a continuación:

16 "Quand à la suite d'une transformation morphologique, une forme subit la différentiation la forme nouvelle corresponde à sa fonction primaire (de fondation), la forme ancienne est réservée pour la fonction secondaire (fondée)" (Kuryłowicz, 1949, p. 30). 


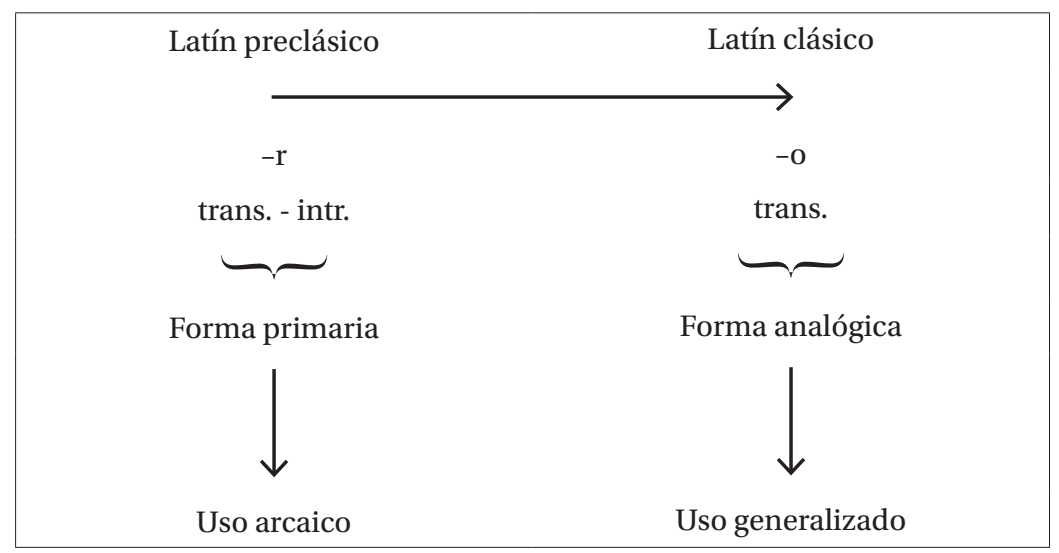

Esquema 1. Explicación diacrónica de verbos deponentes activizados.

Fuente: Elaboración propia.

Si bien esta investigación se centró en el análisis morfosintáctico de tres verbos particulares que, debido a las características propias del grupo, estas particularidades no pueden ser generalizadas a otros verbos que no sean los aquí estudiados, sería interesante abordar, a partir de este acercamiento de cambio analógico, otros subdominios de la experiencia y/u otras categorías semánticas (verbos de dicción, verbos de movimiento, etc.) con el fin de determinar si es posible encontrar una tendencia general de cambio. Después de todo, y en línea con Traugott (2015), el propósito de explicar los cambios de construcción radica no solo en mostrar cómo cambian ciertas formas, sino también en desarrollar un modelo general de lo que es el cambio lingüístico.

\section{Referencias bibliográficas}

Álvarez, O. (2009). Acusativo. En J. M. Baños (Coord.), Sintaxis del latín clásico (pp. 131-154). Madrid: Liceus E-Excellence.

Baerman, M. (2007). Morphological typology of deponency. En M. Maerman, G. G. Corbett, D. Brown, \& A. Hippisley (Eds.), Deponency and Morphological Mismatches (pp. 1-9). London: British Academy.

Baldi, P. (1973). Deponents and middle in Latin. New York: University of Rockester. 
Baños, J. M. (2009). Persona, número y voz. En J. M. Baños (Coord), Sintaxis del latín clásico (pp. 375-404). Madrid: Liceus EExcellence.

Dahl, E. \& Fedriani, C. (2012). The argument structure of experience: Experiential constructions in early Vedic, Homeric Greek and early Latin. Transactions of the Philological Society, 110(3), 342-362. https://doi.org/10.1111/j.1467-968x.2012.01313.x

Dowty, D. (1991). Thematic proto-roles and argument selection. Language, 67(3), 547-619. https://doi.org/10.1353/lan.1991.0021

Elvira, J. (1998). El cambio analógico. Madrid: Gredos.

Fedriani, C. (2014). Experiential constructions in Latin. Series Brill's Studies in Historical Linguistics, vol. 3. Recuperado de https://brill.com/view/title/24526

Flobert, P. (1975). Les verbes déponents latins: des origines à Charlemagne (vol. 17). Paris: Les Belles lettres.

García-Hernández, B. (1990). Transitividad, intransitivación y causas de su desarrollo en latín tardío. Revista Española de Lingüistica, 20(1), 1-16. Recuperado de https://dialnet.unirioja.es/ descarga/articulo/41241.pdf

Grestenberger, L. (julio, 2015). Deponency as reanalysis: A diachronic account of voice mismatches. Trabajo presentado en la 22nd International Conference on Historical Linguistics, Naples.

Grestenberger, L. (2014). Feature mismatch deponency in Indo-European languages. Harvard: Harvard University Press.

Hoffmann, T. \& Trousdale, G. (Eds.). (2013). The Oxford handbook of construction grammar. Oxford: Oxford University Press.

Hopper, P. \& Tompson, S. (1980). Transitivity in grammar and discourse. Language, 56(2), 251-299. https://doi.org/10.1353/ lan.1980.0017

Kuryłowicz, J. (1949). La nature des procès dits "analogiques". Acta linguistica, 5(1), 15-37. https://doi.org/10.1080/03740463.1945 .10410880

Luraghi, S. \& Parodi, C. (2008). Key terms in syntax and syntactic theory. London: Continuum.

Luraghi, S. (2004). Null objects in Latin and Greek and the relevance of linguistic typology for language reconstruction. En Proceedings of the 15th Annual UCLA Indo-European Conference. JIES, The Journal of Indo-European Studies, Monograph, 49, 
234-256. Recuperado de https://www.jies.org/docs/monoseries.html

Luraghi, S. (2010). Experiencer predicates in Hittite. En R. I. Kim, E. Riecken, N. Oettinger, \& M. J. Weiss (Eds.), Ex Anatolia lux (pp. 249-264). Ann Arbor: Beech Stave Press.

Næss, Å. (2007). Prototypical transitivity (Vol. 72). Amsterdam \& Philadelphia: John Benjamins Publishing Company.

Pieroni, S. (2000). Agents in Latin impersonal passives. Mnemosyne, 53(3), 288-301. https://doi.org/10.1163/156852500510606

Pinkster, H. (2015). The Oxford Latin syntax (Vol. 1). Oxford: Oxford University Press.

Torrego, M. E. (octubre, 2010). Función textual y literaria de la pasiva en la Eneida de Virgilio. Trabajo presentado en Perfiles de Grecia y Roma. Actas del XII Congreso Español de Estudios Clásicos, Valencia.

Traugott, E. C. (2008). Grammaticalization, constructions and the incremental development of language: Suggestions from the development of degree modifiers in English. Recuperado de https://web.stanford.edu/ traugott/resources/TraugottEckardtProofs.pdf

Traugott, E. C. (2015). Toward a coherent account of grammatical constructionalization. En J. Barðdal, E. Smirnova, L. Sommerer, \& S. Gildea (Eds.), Diachronic construction gramar (pp. 51-80). Amsterdam: John Benjamins Publishing Company.

Tsunoda, T. (1985). Remarks on transitivity. Journal of Linguistics, 21(2), 385-396. https://doi.org/10.1017/s0022226700010318

Tsunoda, T. (1999). Transitivity and intransitivity. Journal of Asian and African Studies, 57, 1-9. Recuperado de http://repository. tufs.ac.jp/bitstream/10108/21852/1/jaas057001.pdf

Tur, C. (2013). Las construcciones de miseret y de misereor/miseror en latín. Cuadernos de Filología Clásica, Estudios Latinos, 33(2), 239-251. https://doi.org/10.5209/rev_cfcl.2013.v33. n2.43564

Verhoeven, E. (2007). Experiential constructions in Yucatec Maya. Amsterdam: John Benjamins Publishing Company. 